\title{
Apollon : le laser de l'extrême
}

François Amiranoff ${ }^{(1)}$ (francois.amiranoff@polytechnique.fr) et toute l'équipe de projet CILEX-Apollon ${ }^{(2)}$ (1) LULI - CNRS, École polytechnique, CEA : Université Paris-Saclay ; UPMC Université Paris 6 : Sorbonne Universités - 91128 Palaiseau Cedex

(2) Composé de membres de onze laboratoires et institutions du Plateau de Saclay : CPhT, LAL, LASERIX, LCFI0, LIDyL, LLR, LOA, LPGP, LULI, LUMAT, SOLEIL, qui font partie de I'Université Paris-Saclay et dont l'ensemble des tutelles sont : CEA, CNRS, École polytechnique, ENSTA-ParisTech, IOGS, UPMC - Sorbonne Universités, Université Paris-Sud.

Étudier les plasmas relativistes et l'interaction lumière-matière dans des conditions extrêmes, produire de nouvelles sources intenses et brèves de particules et de rayonnement pour une large gamme d'applications, interagir avec les particules

virtuelles du vide et atteindre le régime de l'électrodynamique quantique en champ fort, tels sont les défis d'Apollon, une nouvelle infrastructure de recherche laser

en construction sur le Plateau de Saclay.

D’une puissance inégalée allant jusqu'à 10 pétawatts (PW), les impulsions énergétiques et brèves délivrées par le faisceau principal d'Apollon, jusqu'à 150 joules en 15 femtosecondes (fs), permettront à une large communauté de se lancer dans de nouvelles recherches, aux frontières des connaissances actuelles.

\section{Les différents types de lasers}

Depuis la démonstration du premier laser en 1960, la famille des lasers n'a cessé de s'agrandir, offrant des outils extrêmement variés pour la vie courante, l'industrie et la recherche. Les lasers peuvent être classés en deux types, continus (qui délivrent un faisceau continu de lumière) et impulsionnels. La famille des lasers continus comprend notamment ceux à semi-conducteurs utilisés dans les lecteurs de CD et de DVD, les pointeurs lasers ou les lecteurs de code-barres, d'une puissance de l'ordre $\mathrm{du}$ milliwatt $(\mathrm{mW})$, des lasers de quelques watts (W) que l'on voit souvent dans des soirées animées, et des lasers dans la gamme du kilowatt $(\mathrm{kW})$, voire même du mégawatt (MW), utilisés dans l'industrie pour couper, souder et traiter un grand nombre de matériaux.

Les lasers impulsionnels délivrent de brèves impulsions et peuvent atteindre de très fortes puissances. Ils permettent entre autres d'étudier des phénomènes brefs. Fonctionnant initialement dans le régime milliseconde (ms) ou microseconde $(\mu \mathrm{s})$, le développement de techniques nouvelles, comme le blocage de modes, a permis de passer à des lasers fonctionnant dans le régime nanoseconde (ns), puis, grâce à la méthode d'amplification d'impulsions à dérive de fréquence développée dans les années 1980 (CPA pour Chirped Pulse Amplification) [2] (fig. 1), dans le régime picoseconde $\left(1 \mathrm{ps}=10^{-12} \mathrm{~s}\right)$ puis femtoseconde (1 fs $\left.=10^{-15} \mathrm{~s}\right)$.

Dans le cadre de la recherche, l'outil laser idéal dépend largement des milieux que l'on souhaite produire et étudier. En se limitant aux lasers délivrant des impulsions de forte énergie, deux types d'impulsions sont utilisés : les impulsions «longues" (ns) et les brèves (ps, fs).
Pour reproduire en laboratoire des milieux chauds et denses (cœurs d'étoiles, noyaux planétaires, jets astrophysiques...) ou pour la production d'énergie par fusion thermonucléaire, une énergie élevée et des impulsions longues (ns) sont nécessaires. Dans ce domaine, le laser le plus énergétique est le NIF (National Ignition Facility) aux USA, qui sera progressivement rejoint par le Laser MégaJoule (LMJ) en France. Il délivre des impulsions allant jusqu'à 3 mégajoules (MJ) pour des impulsions d'une dizaine de ns, soit une puissance de l'ordre de 0,3 PW (1 PW = $\left.10^{15} \mathrm{~W}\right)$. D'une taille équivalente à la Tour Eiffel et regroupant plus d'une centaine de faisceaux individuels de $40 \mathrm{~cm}$ de diamètre, ces installations ne délivrent qu'un à quelques tirs par jour. Une expérience consiste le plus souvent à focaliser ces impulsions à l'aide de lentilles ou de miroirs sur des petites cibles de quelques $\mu \mathrm{m}$ à quelques $\mathrm{mm}$. Un paramètre important est l'intensité lumineuse au niveau de la cible. En régime ns, les intensités utilisées dépassent rarement $10^{16} \mathrm{~W} / \mathrm{cm}^{2}$, permettant toutefois d'obtenir des températures au niveau de la cible de plusieurs millions de degrés, comparables à celle du centre du Soleil. La puissance et la qualité des faisceaux produits en régime d'impulsions brèves sont telles que les intensités obtenues après focalisation atteignent aujourd'hui quelques $10^{19} \mathrm{~W} / \mathrm{cm}^{2}$ en régime ps et quelques $10^{21} \mathrm{~W} / \mathrm{cm}^{2}$ en régime $\mathrm{fs}$. À ces intensités, l'interaction entre une impulsion laser et une cible produit en particulier des sources intenses et brèves de particules énergétiques et de rayonnement (voir p. 63), dont certaines sont utilisées pour sonder les plasmas formés en régime ns, fournissant ainsi une 

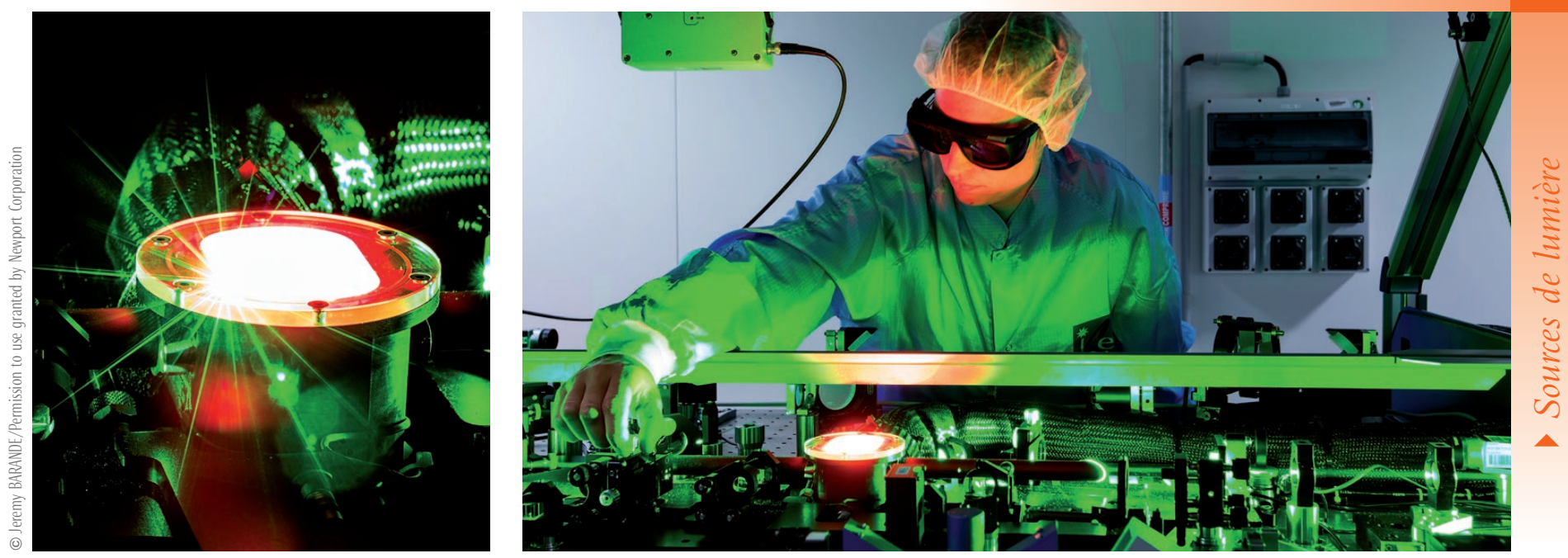

Salle pilote du laser Apollon, où a lieu son amplification.

excellente résolution temporelle à l'échelle ps. C'est une des raisons pour lesquelles ont été développés des lasers ps délivrant des énergies par impulsion de l'ordre de plusieurs kJ, soit une puissance de l'ordre du PW. Le laser PETAL, couplé au LMJ, délivrera des impulsions de 3,5 kJ en régime ps, faisant du couple LMJ-PETAL un instrument unique pour la recherche académique. Le LULI, infrastructure de recherche nationale et européenne, dispose de deux installations couplant des impulsions ns et $\mathrm{ps, \text {dont }}$ LULI2000 qui produit des impulsions de $1 \mathrm{~kJ}$ en régime ns couplées à des impulsions de $100 \mathrm{~J}$ en régime ps.

En régime fs, les matériaux et les optiques utilisés limitent les énergies par impulsion à des valeurs plus modestes. En France, le LOA (à Palaiseau) et le LIDyL (au CEA/Saclay) disposent d'installations produisant des impulsions d'énergie de l'ordre du joule en 30 à 50 fs (soit environ $100 \mathrm{TW}$, ou $10^{14} \mathrm{~W}$ ). Dans le monde, de nombreuses installations fonctionnent à des puissances semblables, ou commencent à atteindre le régime $\mathrm{PW}$.

\section{Le projet Apollon}

Le laser Apollon se distingue des précédents par sa puissance plus élevée (jusqu'à $10 \mathrm{PW}$ ), en régime fs. Il délivrera des impulsions de $150 \mathrm{~J}$ en 15 fs. Il précédera les installations européennes ELI (Extreme Light Infrastructure) en République tchèque, Roumanie et Hongrie, de puissance comparable.

Comme tous les lasers énergétiques en impulsions brèves, Apollon utilise la technique CPA (fig. 1), avec des ajouts spécifiques pour maintenir une très large bande spectrale après amplification,
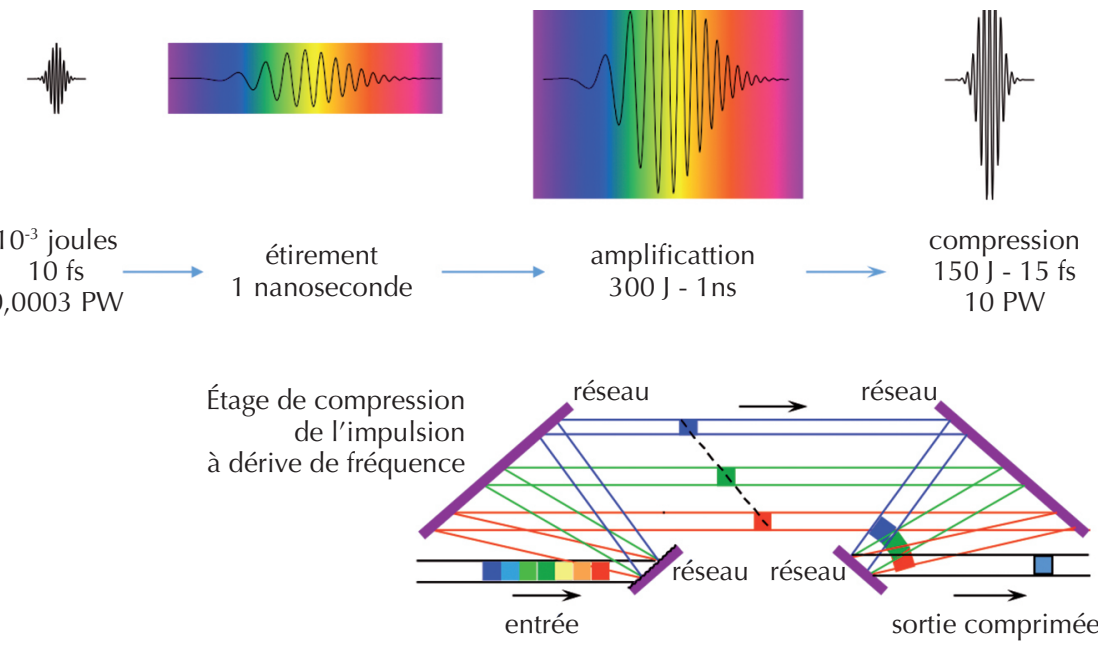

1. Schéma de principe de la chaîne Apollon, basée sur la technique CPA.

Une impulsion brève et peu intense est produite dans un oscillateur commercial ( $25 \mathrm{fs}, 1,5 \mathrm{~mJ})$. Pour atteindre le régime sub-10 fs, son spectre en fréquence est élargi par effet non linéaire à la traversée d'une cellule de gaz. Ce faisceau est ensuite étiré et amplifié en deux étapes. Lors d'une première étape, l'étirement est produit par un filtre acousto-optique jusqu'à plusieurs ps et l'amplification est obtenue par la technique OPCPA (pour Optical Parametric Chirped Pulse Amplification - Amplification Paramétrique d'Impulsions à Dérive de Fréquence), qui consiste à coupler dans un cristal non linéaire, l'impulsion à amplifier avec un faisceau de pompe ps spécialement développé à cet effet dans le cadre du projet. À la sortie de l'étage d'amplification ps, l'impulsion a un spectre suffisamment large pour des impulsions sub-10 fs (de $700 \mathrm{~nm}$ à $900 \mathrm{~nm}$ ) et une énergie de l'ordre du mJ (en haut à gauche). L'étirement jusqu'au régime ns est obtenu par un système à réseaux de diffraction, le principe étant de faire parcourir des trajets distincts aux différentes longueurs d'onde composant le faisceau. Ce principe est illustré dans le cas de la géométrie (différente) utilisée pour la compression des impulsions amplifiées.

Pour limiter l'intensité lumineuse au niveau des optiques, le faisceau final, d'un diamètre de 140 mm est élargi jusqu'à $400 \mathrm{~mm}$ et comprimé jusqu'au régime femtoseconde par un système à quatre réseaux de grande taille $(900 \mathrm{~mm} \times 450 \mathrm{~mm})$. Le faisceau est diffracté par un premier réseau. Chaque longueur d'onde suit un trajet distinct jusqu'à un second réseau où les différentes composantes sont de nouveau parallèles. Un deuxième étage identique double l'effet de compression déjà obtenu. Dans cette phase de compression, les photons « rouges", à l'avant de l'impulsion en entrée, effectuent un trajet plus long que les photons « bleus ». En régime ns, l'amplification est obtenue d'abord par un système $\mathrm{OPCPA}$ analogue au précédent avec un faisceau de pompe ns spécifique, puis dans des amplificateurs classiques à cristaux de Ti:Sa (titane-saphir) pompés par des faisceaux de pompe ns commerciaux à verre au néodyme doublés en fréquence à 0,53 $\mu \mathrm{m}$. 


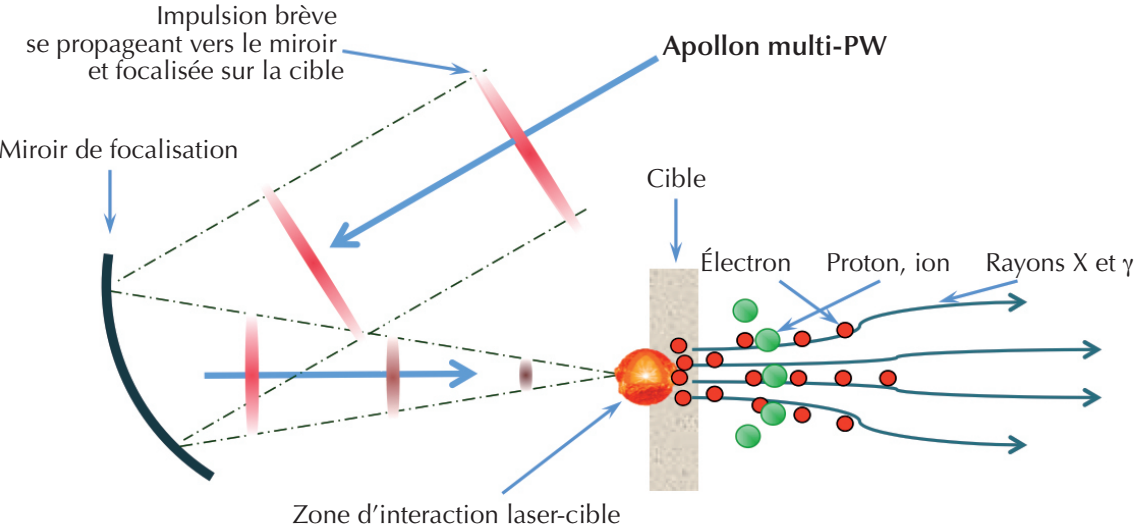

2. L'impulsion multi-PW d'Apollon consiste en un mince disque de photons de $400 \mathrm{~mm}$ de diamètre et 5 microns d'épaisseur. Elle est focalisée par une parabole hors-axe dans une tache focale de quelques microns de diamètre. L'ensemble de l'énergie est alors concentré dans un cube de quelques microns de côté. L'intensité au niveau du point de focalisation dépasse $10^{22} \mathrm{~W} / \mathrm{cm}^{2}$. À cette intensité, une grande partie de l'énergie incidente est transférée à des électrons relativistes qui, à leur tour, conduisent à l'accélération de faisceaux d'ions et à la production de rayonnement jusqu'aux rayons gamma. Cependant, si la cible est chauffée, même à des températures modestes de quelques eV (quelques dizaines de milliers de ${ }^{\circ}$ ), avant l'arrivée de l'impulsion principale par des préimpulsions qui n'auraient pas été supprimées, sa surface se détend et l'interaction se fait avec un plasma de faible densité plutôt qu'avec la cible solide initiale.

\section{$\gg \gg$}

permettant de « recomprimer » les impulsions jusqu'à 15 fs. Le schéma de principe de cette technique et les paramètres des impulsions d'Apollon [1] sont résumés dans la figure 1. Amplifier directement une impulsion brève à haute énergie dans un milieu matériel solide est, de fait, impossible. L'intensité lumineuse au cours de la propagation dans l'air et dans les matériaux serait si élevée, qu'elle induirait des effets non linéaires qui détruiraient les qualités optiques du faisceau, et endommagerait les matériaux eux-mêmes. La technique consiste donc à étirer une impulsion brève avant de l'amplifier, puis à la recomprimer... La puissance finale atteindra alors les $10 \mathrm{PW}$, correspondant à $150 \mathrm{~J}$ en $15 \mathrm{fs}$, soit seulement quelques cycles optiques. Le taux de répétition sera de 1 tir/minute, de telle sorte que, malgré la puissance extrême de chaque impulsion, la puissance moyenne d'Apollon ne sera que de 2,5 W!

Obtenir de telles impulsions intenses et brèves représente un défi scientifique et technologique de grande ampleur qui n'a encore jamais été réalisé à cette échelle. Il s'agit en effet de construire une chaîne qui maintient une bande spectrale très large $(200 \mathrm{~nm})$, tout en amplifiant à forte énergie (300 J) un faisceau de grand diamètre $(140 \mathrm{~mm})$, et qui maintient en même temps une excellente qualité spatiale et temporelle. Pour les expériences, l'impulsion est focalisée par un miroir parabolique sur de petites cibles solides ou gazeuses (fig. 2). Tous les défauts du faisceau, principalement les défauts de phase spatiale et temporelle, vont se traduire par une tache focale dégradée, c'est-à-dire plus grande et moins régulière que la petite tache de quelques microns de diamètre obtenue pour un faisceau parfait, et par des effets temporels d'allongement et de déformation. Ces effets diminuent de façon significative l'intensité maximum que l'on obtient au foyer du système de focalisation, qui, dans le cas d'Apollon doit dépasser $10^{22} \mathrm{~W} / \mathrm{cm}^{2}$.

Un autre défi est de s'affranchir de toute préimpulsion qui pourrait irradier la cible avant l'arrivée du pic d'intensité. En effet, le seuil d'endommagement d'une cible solide, inférieur à, ou de l'ordre de $10^{10} \mathrm{~W} / \mathrm{cm}^{2}$, est $10^{12}$ fois plus faible que l'intensité maximum. Or, que ce soit dès le départ au niveau de l'oscillateur, dans la phase d'amplification dans le régime ns ou à cause d'effets non linéaires dans les milieux traversés, une impulsion "standard " présente un contraste typique entre les préimpulsions et l'impulsion principale de l'ordre de seulement $10^{7}$. Comme dans d'autres installations de lasers de puissance, des systèmes sont implantés au début de la chaine pour supprimer au mieux ces préimpulsions et atteindre un contraste de l'ordre de $10^{10}$. Pour améliorer encore ce contraste, il est désormais courant d'utiliser des miroirs plasma. Constitués d'une optique transparente sur laquelle le faisceau est dirigé bien avant le point de focalisation maximum, ils laissent passer la partie initiale du faisceau, en-dessous du seuil d'endommagement, et réfléchissent la partie la plus intense dès qu'un plasma commence à se former à la surface de l'optique. C'est cette partie réfléchie du faisceau qui interagit alors avec la cible située en aval. Bien réglé, un miroir plasma simple permet d'améliorer le contraste d'un facteur proche de 100 , et un miroir double (deux miroirs l'un après l'autre) d'un facteur compris entre $10^{3}$ et $10^{4}$.

Au-delà des amateurs de records, pour le physicien des plasmas, la puissance extrême délivrée par une chaîne laser est surtout intéressante pour l'intensité maximale obtenue au point de focalisation. C'est elle qui détermine le régime d'interaction laser-cible et les paramètres obtenus dans le plasma. L'énergie totale par impulsion et la durée de celle-ci sont les deux autres paramètres qui déterminent les expériences qui peuvent être réalisées. Disposer d'impulsions de forte énergie permettra par exemple d'accélérer des électrons sur de grandes distances, ou de produire des faisceaux intenses contenant un grand nombre de protons ou de rayons $\mathrm{X}$. La brièveté des impulsions laser permettra de plus de produire des sources de rayonnement encore plus brèves, jusque dans le domaine attoseconde $\left(1\right.$ as $\left.=10^{-18} \mathrm{~s}\right)$. Les intensités extrêmes obtenues sur la cible donneront accès à de nouveaux régimes d'interaction, permettant même d'étudier, dans les plasmas ou dans le vide, des problèmes d'électrodynamique en champ fort. Ces différents thèmes sont détaillés ci-dessous.

Sur le site d'Apollon, les expériences seront réalisées dans deux salles. La salle "longue focale " sera plus spécifiquement dédiée aux interactions sur de grandes longueurs, en utilisant des miroirs de longueur focale de plusieurs mètres à plusieurs dizaines de mètres. L'autre salle, au contraire, sera dédiée aux interactions à haute intensité, en utilisant des longueurs focales beaucoup plus courtes, de l'ordre du mètre, soit des taches focales de quelques microns de diamètre. Dans le premier cas, l'intensité maximum est plus faible mais se maintient sur plusieurs centimètres, et sur seulement quelques dizaines de microns dans le second cas. 


\section{Accélération d'électrons}

Contrairement aux structures accélératrices classiques, les plasmas chauds, déjà totalement ionisés, peuvent supporter des champs électriques extrêmement élevés, uniquement limités par le nombre de charges électriques présentes. De plus, le mouvement combiné des électrons dans les champs électrique et magnétique d'un faisceau laser tend à les repousser vers les zones de champ faible, produisant ainsi une séparation de charge oscillant à la fréquence naturelle des électrons dans le milieu : la fréquence plasma. Le champ électrique associé à cette séparation de charge atteint facilement des valeurs de l'ordre de ou supérieures à $10^{12} \mathrm{~V} / \mathrm{m}$ et se propage à la vitesse du faisceau laser, très proche de celle de la lumière pour des densités électroniques faibles. Un électron injecté ou piégé dans ce champ, et dont la vitesse est également proche de celle de la lumière, peut alors être accéléré sur de grandes longueurs [3]. Le record actuel est proche de $4 \mathrm{GeV}$ et a été obtenu sur un plasma de l'ordre du centimètre de longueur.

Un schéma en deux étapes est proposé dans la figure 3. Avec Apollon, l'énergie des électrons pourrait atteindre $10 \mathrm{GeV}$ pour une longueur d'accélération de l'ordre de $10 \mathrm{~cm}$, beaucoup plus grande que la zone de quelques centaines de $\mu \mathrm{m}$ de longueur représentée sur la figure 3 .

Un des objectifs d'Apollon est de démontrer la pertinence d'un tel schéma et la possibilité de le contrôler de façon fiable et répétitive [4]. Ce schéma pourrait ensuite évoluer vers des énergies encore plus élevées en ajoutant des étages accélérateurs supplémentaires.

\section{Accélération de protons, d'ions}

Selon le même principe, plusieurs schémas de production par laser de jets intenses de protons et d'ions énergétiques ont été proposés ; certains se sont révélés accessibles avec les lasers actuels [5].

À faible intensité laser, le mouvement d'un électron dans les champs $\mathbf{E}$ et $\mathbf{B}$ se produit principalement dans la direction de $\mathbf{E}$ (vertical dans la figure 3) sous l'effet de la force $q \mathbf{E}$, perpendiculaire à la direction de propagation du faisceau. Lorsque l'intensité augmente, la vitesse de l'électron s'approche de celle de la lumière $^{(1)}$ et la force $\mathrm{q}(\mathbf{v} \times \mathbf{B})$ (où $\mathrm{B} \approx \mathrm{E} / \mathrm{c}$

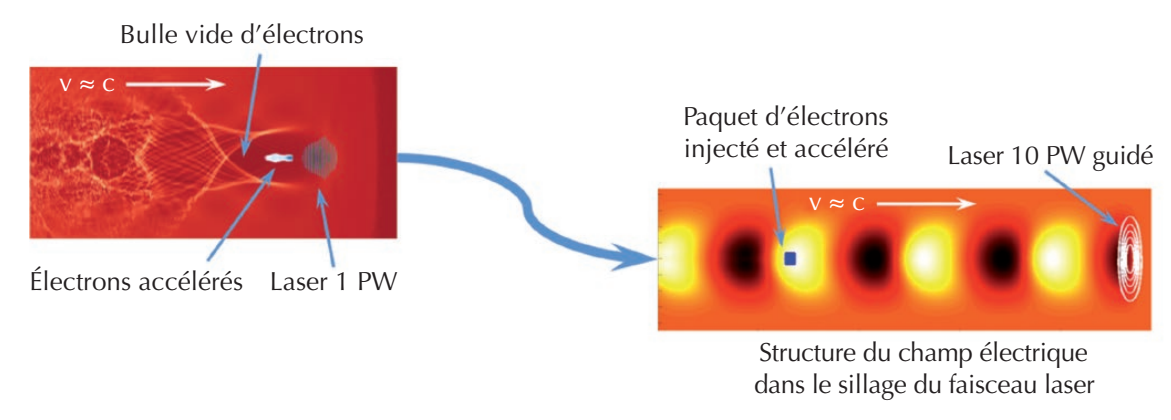

\section{Accélération d'électrons en deux étages.}

Un faisceau d'électrons de plusieurs dizaines de MeV est produit par interaction entre un faisceau laser de 1 PW et un jet de gaz. L'image de gauche est le résultat d'une simulation numérique et représente la densité électronique dans le plasma, créé par l'ionisation du gaz par le laser, dans le sillage de l'impulsion laser qui se dirige vers la droite. Le champ laser expulse la plus grande partie des électrons, créant ainsi une " bulle » vide d'électrons. Une faible partie de ces électrons peut toutefois rentrer dans la bulle (juste à gauche de la flèche «bulle vide d'électrons ») après en avoir été expulsée, et être accélérée en profitant du champ électrique de charge d'espace qui y règne.

Après leur sortie du premier étage, ces électrons sont guidés par des éléments magnétiques vers le deuxième étage (à droite) qui fonctionne dans un régime d'intensité plus faible. Le faisceau de 10 PW est focalisé dans un tube capillaire rempli de gaz (non visible sur la figure) où il excite une onde plasma, visible ici par l'alternance de zones positives et négatives du champ électrique. Cette onde accélère les électrons injectés sur une grande longueur pouvant aller jusqu'à plusieurs dizaines de centimètres, avant d'atteindre une zone où le champ changerait de signe et le ralentirait. On peut voir l'impulsion laser à droite, le champ électrique alternatif dans son sillage et le paquet d'électrons (en bleu) qui à été injecté et accéléré.

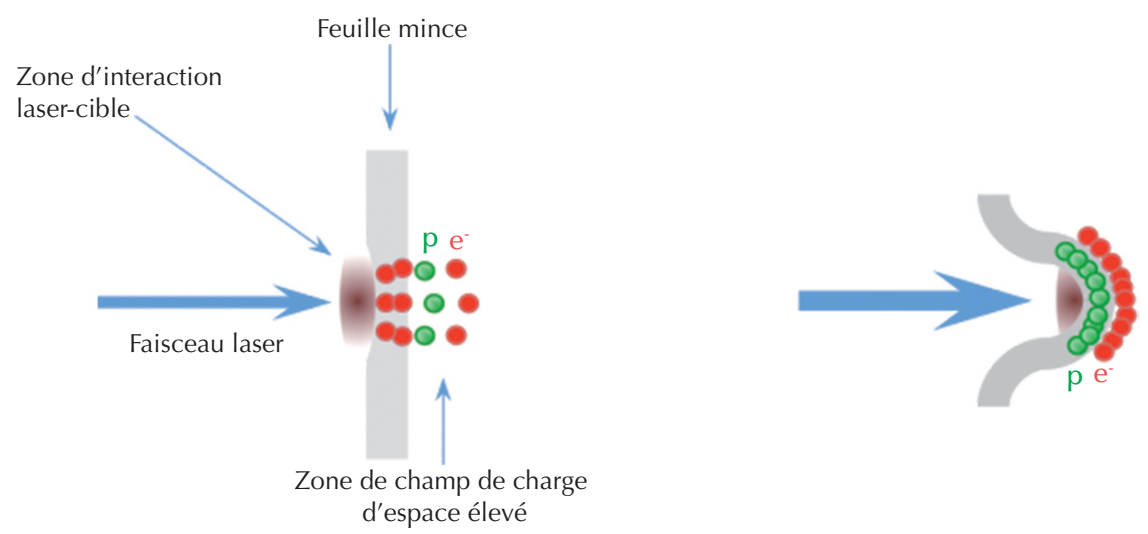

\section{Accélération de protons.}

À gauche, à intensité laser modérée, par exemple $10^{18} \mathrm{~W} / \mathrm{cm}^{2}$, une petite partie des électrons dans la zone d'interaction est accélérée à haute énergie (points rouges) et sort au niveau de la face arrière de la feuille mince. II s'établit alors un champ de charge d'espace entre ces électrons et l'arrière de la cible chargé positivement. Ce champ retient les électrons éjectés et accélère les protons (points verts) proches de la face arrière. L'énergie maximum ainsi obtenue aujourd'hui est de l'ordre de $100 \mathrm{MeV}$.

À droite, à plus haute intensité, par exemple $10^{23} \mathrm{~W} / \mathrm{cm}^{2}$, l'ensemble des électrons de la feuille est poussé vers l'avant. Le champ électrique de charge d'espace, beaucoup plus élevé que dans le cas précédent, devrait accélérer la totalité des ions de la feuille proches de la tache focale laser à des énergies pouvant atteindre plusieurs GeV. 
\〉

dans le vide) devient du même ordre que qE. Les électrons sont alors principalement accélérés vers l'avant, dans la direction du faisceau laser. La figure 4 montre deux cas limites qui en découlent pour l'accélération d'ions. Les champs électriques de charge d'espace à l'avant de la cible sont déterminés par l'énergie des électrons $(\approx \mathrm{MeV})$ divisée par la distance moyenne à laquelle ceux-ci peuvent s'écarter de la cible $(\approx \mu \mathrm{m})$ avant d'être arrêtés par le champ électrique. Même dans le cas d'intensités modérées $\left(\approx 10^{18} \mathrm{~W} / \mathrm{cm}^{2}\right)$, ils atteignent des valeurs typiques de l'ordre du $\mathrm{MV} / \mu \mathrm{m}$.

Avec Apollon, à des intensités dépassant les $10^{22} \mathrm{~W} / \mathrm{cm}^{2}$, il devrait être possible d'accélérer des faisceaux de protons jusqu'à des énergies de l'ordre du GeV.
La présence de faisceaux intenses d'électrons ou d'ions de forte énergie permet également de produire d'autres types de faisceaux intenses de particules, comme des faisceaux de neutrons ou des jets de paires électron-positron. Ceux-ci peuvent être générés directement dans la cible initiale, ou dans une cible secondaire (fig. 5). Grâce à la puissance du faisceau laser initial, les faisceaux secondaires sont à la fois très brefs et très intenses.

Il sera possible de générer des neutrons de plusieurs dizaines de $\mathrm{MeV}$ et des faisceaux d'électrons-positrons suffisamment denses pour être pertinents pour des études relatives à l'astrophysique.

\section{Faisceaux de rayons X et gamma}

Lors de l'interaction entre un faisceau laser intense et une cible solide, de nombreux mécanismes sont susceptibles de générer des faisceaux intenses de rayons $\mathrm{X}$. En raison du champ électrique élevé du faisceau laser, le déplacement des électrons de la cible est fortement non linéaire, impliquant la fréquence du laser et de nombreuses harmoniques. Le rayonnement généré par le mouvement de ces électrons contient des harmoniques de la fréquence laser jusqu'à des ordres extrêmement élevés, jusque dans le domaine des rayons $\mathrm{X}$, au $\mathrm{keV}$ et audelà (2). C'est le principe du miroir oscillant relativiste, présenté en figure 6.
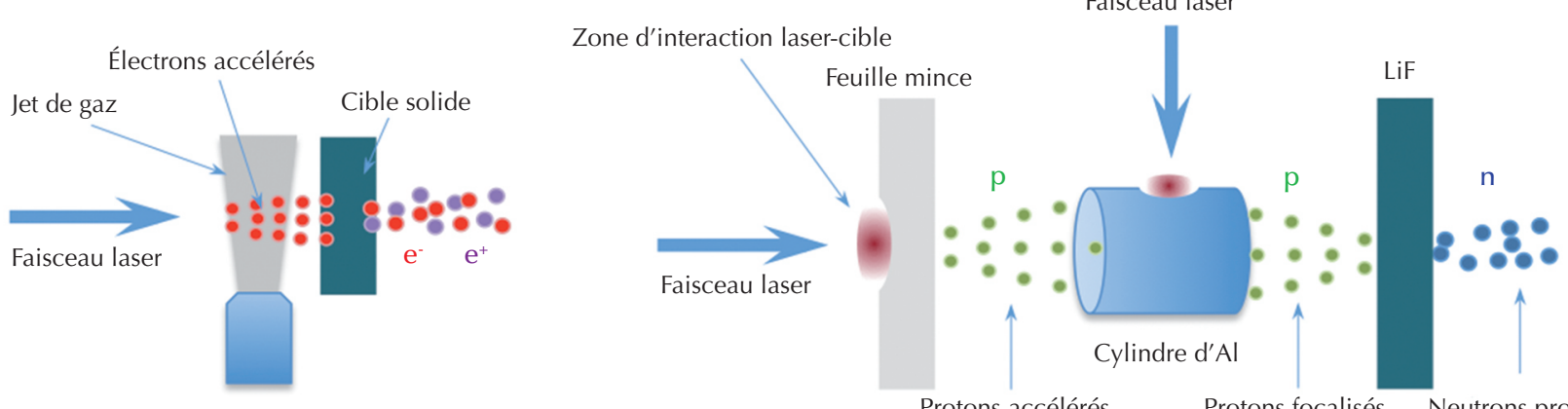

Protons accélérés

Protons focalisés

utrons produits dans LiF

5. Production de particules secondaires à partir de faisceaux primaires.

À gauche, un faisceau d'électrons accéléré dans un jet de gaz produit, dans une cible solide placée près de la sortie du jet, un ensemble de paires électron-positron $\left(e^{-}-e^{+}\right)$, soit directement par interaction avec le champ atomique, soit par l'intermédiaire de rayons gamma, également produits dans le matériau.

À droite, un faisceau de protons accéléré à partir d'une cible mince (comme sur la figure 4) est focalisé par une lentille électrique déclenchée par un faisceau laser annexe. En effet, comme pour la cible plane, un champ de charge d'espace est généré sur la face arrière de la cible, c'est-à-dire ici sur la face interne du cylindre. Ce faisceau de protons produit des neutrons par réactions nucléaires dans la cible de LiF, par exemple : p + ${ }^{7} \mathrm{Li} \rightarrow{ }^{7} \mathrm{Be}+\mathrm{n}$.
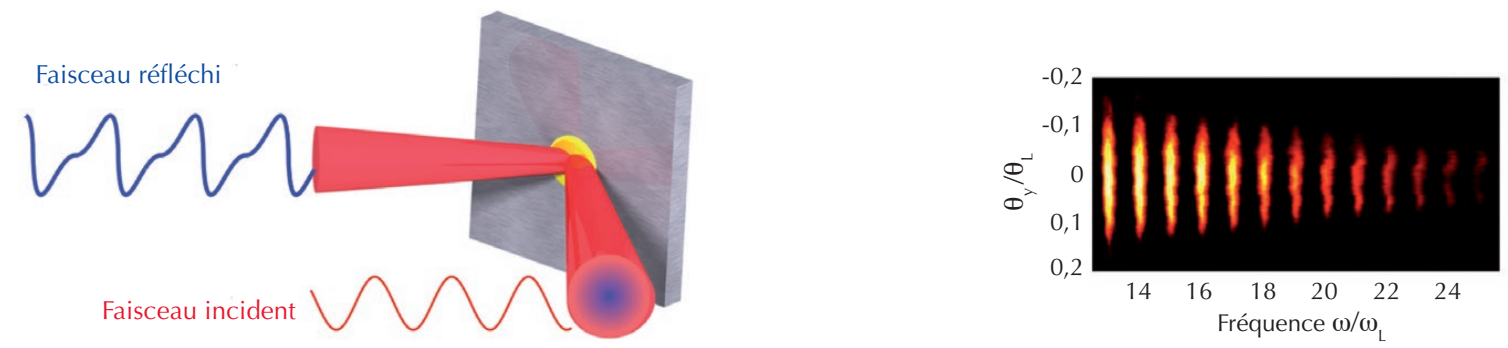

6. Principe du miroir oscillant relativiste. Le champ électrique du faisceau incident, représenté ici constant sur quelques périodes, fait osciller la surface de la cible selon l'axe du faisceau à la fréquence du laser et à des vitesses proches de la vitesse de la lumière. Le mouvement de la surface n'est plus du tout sinusoïdal, mais présente des évolutions extrêmement rapides entre une vitesse nulle et une vitesse proche de celle de la lumière. La lumière laser réfléchie par ce miroir oscillant relativiste contient alors un spectre très large de fréquences (image de droite, obtenue à une intensité de l'ordre de $10^{19} \mathrm{~W} / \mathrm{cm}^{2}$ ). 
Avec Apollon, on peut espérer obtenir plusieurs centaines d'harmoniques, donc des photons dans le domaine des rayons $\mathrm{X}$. L'ensemble de ces harmoniques étant cohérent, le faisceau réfléchi permet d'obtenir des durées d'impulsions bien plus brèves que celle du faisceau initial. Le nombre d'harmoniques, et donc la largeur totale du spectre émis, étant une fonction croissante de l'intensité laser, l'intensité extrême d'Apollon devrait permettre d'obtenir des faisceaux plus brefs que le record actuel, proche de 60 attosecondes.

Disposant de deux faisceaux intenses, il sera possible avec Apollon de générer des faisceaux $\mathrm{X}$ et gamma, en irradiant à haute intensité un faisceau d'électrons du domaine du $\mathrm{GeV}$ produit par un premier faisceau (fig. 7). La diffusion Compton des photons laser sur les électrons relativistes produit un rayonnement de fréquence maximum $4 \gamma^{2} v$, où $\gamma$ est le facteur relativiste des électrons $(\gamma \approx 2000$ pour une énergie de $1 \mathrm{GeV}$ ) et $v$ la fréquence du laser. Avec un faisceau d'électrons de $1 \mathrm{GeV}$, il sera ainsi possible de produire un faisceau intense de rayons gamma de plusieurs $\mathrm{MeV}$.

\section{La physique en champ fort}

Les intensités extrêmes délivrées par Apollon vont permettre d'ouvrir un nouveau champ de recherche : la physique en champ fort. L'exemple qui le symbolise le mieux est la création de paires électronpositron dans le vide. Le vide est peuplé en permanence de particules virtuelles, comme des paires électron-positron, qui apparaissent et s'annihilent en des temps de l'ordre de $10^{-21}$ s. Si un champ électrique permet de séparer ces paires d'une distance de l'ordre de la longueur de Compton, c'est-à-dire de la taille caractéristique de leur fonction d'onde $\left(\approx 4 \times 10^{-13} \mathrm{~m}\right)$, avant qu'elles ne s'annihilent, ces paires virtuelles peuvent devenir réelles. Il faut noter que l'énergie de ces particules ne provient pas du vide, mais bien du champ électrique extérieur lui-même. Le champ a en fait fourni une énergie au moins égale à deux fois l'énergie de masse de l'électron, $\mathrm{mc}^{2}$. Ce champ est appelé champ de Schwinger et sa valeur $\approx 1,3 \times 10^{18} \mathrm{~V} / \mathrm{m}$ correspond à une intensité laser proche de $10^{29} \mathrm{~W} / \mathrm{cm}^{2}$. Même si les intensités maximum d'Apollon seront très inférieures à cette valeur, plusieurs schémas permettent malgré tout de tester des effets de champ fort.

Aux intensités qui seront accessibles avec Apollon, les électrons subiront des accélérations telles qu'ils pourront émettre des photons d'énergie proche de celle de l'électron lui-même, faisant alors intervenir des effets significatifs de physique quantique. Les calculs prévoient qu'une très large fraction de l'énergie laser absorbée sera émise sous forme de rayonnement, modifiant ainsi profondément le comportement global du plasma.

Enfin, un électron suffisamment énergétique se propageant vers le paquet de photons qu'est l'impulsion laser, verra, dans son repère propre, un champ multiplié par le facteur relativiste $\gamma$ (défini précédemment), et une intensité multipliée par $\gamma^{2}$. Pour un électron de $1 \mathrm{GeV}, \gamma \cong 2000$ et l'intensité est multipliée par $4 \times 10^{6}$. Une intensité laser, dans le repère du laboratoire, de l'ordre de $10^{22} \mathrm{~W} / \mathrm{cm}^{2}$ est alors suffisante pour atteindre, dans le repère de l'électron, le régime de champ fort. Ce type d'expérience sera tout à fait accessible sur Apollon.

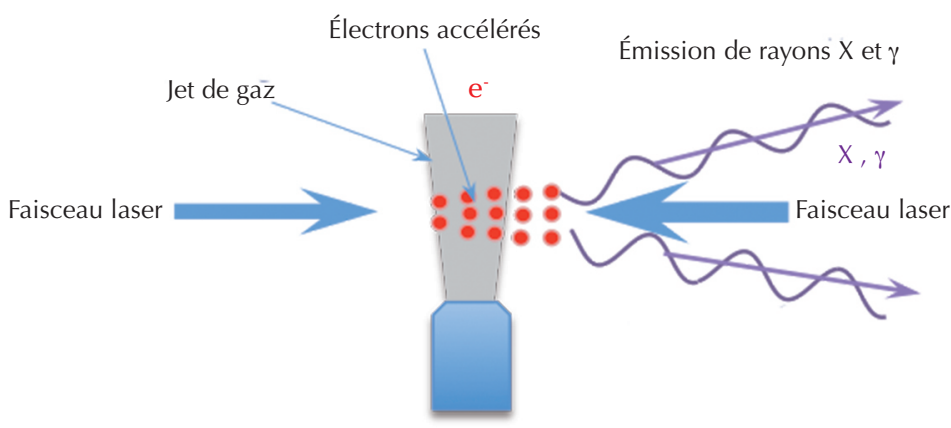

7. Production de rayons $X$ et gamma par diffusion Compton d'un faisceau laser intense sur un faisceau d'électrons accéléré par laser dans un jet de gaz. Le mouvement des électrons dans le faisceau laser venant de la droite produit un rayonnement intense dans les domaines $X$ et gamma.

\section{Conclusion}

Les caractéristiques principales de la nouvelle installation laser Apollon que sont l'énergie, la durée et l'intensité des impulsions, et la présence de plusieurs faisceaux synchronisés, vont permettre d'élargir le domaine d'application des lasers de puissance en ouvrant de nouveaux champs de recherche : de la physique fondamentale, comme la physique en champ fort ou la physique des plasmas relativistes, à des thèmes plus appliqués liés aux nombreuses sources secondaires de particules et de rayonnements qui seront générés sur l'installation. Future "Infrastructure de Recherche nationale et européenne ", Apollon et ses équipes seront bientôt prêts à accueillir une large communauté d'utilisateurs.

(1) Cette condition de forte accélération est obtenue pour I $\lambda^{2} \approx 10^{18} \mathrm{~W} \cdot \mathrm{cm}^{-2} \cdot \mu \mathrm{m}^{2}$, où I et $\lambda$ sont respectivement l'intensité et la longueur d'onde du laser.

(2) Un exemple d'utilisation de ces harmoniques est donné dans la référence [6].

\section{Remerciements}

Sous la responsabilité du laboratoire LULI, le projet Apollon est l'œuvre de l'ensemble de la communauté CILEX (Centre Interdisciplinaire Lumière Extrême), avec des contributions précieuses d'équipes de l'Institut Lasers et Plasmas. Portée par le CNRS avec l'École polytechnique et le CEA, ainsi que l'Institut d'Optique Graduate School (IOGS), l'ENSTA Paris-Tech, l'Université Paris-Sud et l'UPMC, l'installation Apollon se situe au cœur de l'Université Paris-Saclay.

Elle a reçu de nombreux soutiens : contrat ILE 07-CPER017-01 financé par le ministère de la Recherche, le CNRS, la région Île-de-France et le département de l'Essonne, et fonds FEDER européens. De plus, ce travail a bénéficié d'une aide de l'État, gérée par l'Agence Nationale de la Recherche au titre du programme Investissements ANR-10-EQPX-25.

En savoir plus
1• www.apollon-laser.fr ; cilexsaclay.fr .
2• D. Strickland et G. Mourou, Opt. Commun.
$\mathbf{5 6}$ (1985) 218.
3• V. Malka, Physics of Plasmas, 19 (2012) 055501.
4• B. Cros et al., NIMA 740 (2014) 27.
5• M. Borghesi, NIMA 740 (2014) 6.
6・ V. Blanchet, Reflets de la physique 41 (2014) 30.

